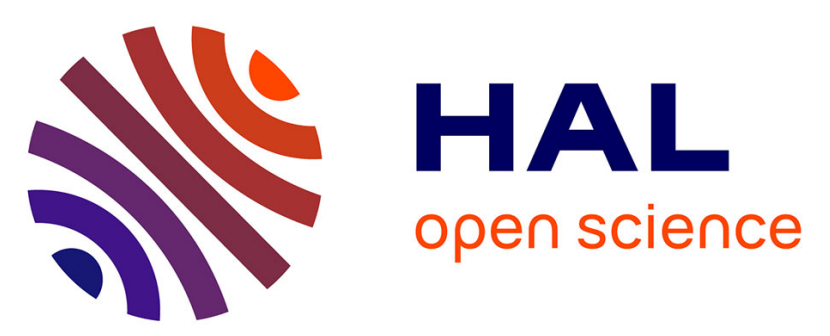

\title{
ARTHUR SCHUSTER. - Ueber das Spectrum des Stickstoffs ( Sur le spectre de l'azote); Annales de Poggendorff, t. CXLVII, p. 106; 1872
}

\author{
A. Cornu
}

\section{- To cite this version:}

A. Cornu. ARTHUR SCHUSTER. - Ueber das Spectrum des Stickstoffs ( Sur le spectre de l'azote); Annales de Poggendorff, t. CXLVII, p. 106; 1872. J. Phys. Theor. Appl., 1873, 2 (1), pp.34-36. 10.1051/jphystap:01873002003401 . jpa-00236877

\section{HAL Id: jpa-00236877 https://hal.science/jpa-00236877}

Submitted on 1 Jan 1873

HAL is a multi-disciplinary open access archive for the deposit and dissemination of scientific research documents, whether they are published or not. The documents may come from teaching and research institutions in France or abroad, or from public or private research centers.
L'archive ouverte pluridisciplinaire HAL, est destinée au dépôt et à la diffusion de documents scientifiques de niveau recherche, publiés ou non, émanant des établissements d'enseignement et de recherche français ou étrangers, des laboratoires publics ou privés. 
ARTHUR SCHUSTER. - Ueber das Spectrum des Stickstoffs (Sur le spectre de l'azote); Annales de Poggendorff, t. CXLVII, p. 106; 1872 .

Plücker et Hittorf ont décrit dans leur Mémoire sur les spectres des gaz ( $\left.{ }^{1}\right)$ les apparences diverses que les variations de température et de pression produisent dans ces spectres, et ont été conduits à distinguer pour un certain nombre d'entre eux deux espèces de spectres. Selon ces physiciens, le spectre du premier ordre correspondant à une basse température serait en général formé de bandes brillantes; celui du second ordre formé de lignes se présenterait au contraire lorsque la pression est très-faible et la température du gaz très-élevée : les exemples les plus frappants sur lesquels s'étayait cette théorie étaient l'hydrogène et l'azote.

Cette manière d'interpréter les faits paraît devoir être entièrement rejetée, et la conclusion infiniment plus satisfaisante pour l'esprit, à savoir que les gaz simples incandescents possèdent un spectre unique, se confirme de plus en plus.

Les recherches chimiques de M. Berthelot et les observations de M. Ångström ont prouvé que le spectre de bandes attribué à l'hydrogène appartient à un composé organique particulier, l'acétylène, très-facile à produire sous l'influence de l'étincelle électrique lorsque l'hydrogène se trouve en contact avec des traces de carbone.

L'hydrogène n'a donc en réalité qu'un seul spectre composé des trois lignes brillantes bien connues qui fournissent au spectre solaire les trois lignes sombres C, F et $h$ du spectre de Frauenhofer.

(') Philosophical Transactions, t. CLV, p. I. 
D'après Ångström ct de 'Thalén, ces radiations ont pour longueur d'onde (exprimée en dix millionièmes de millimètre) :

$$
\begin{aligned}
& \mathbf{C}=656 \mathbf{2} \\
& \mathbf{F}=486 \mathbf{r} \\
& \boldsymbol{h}=4 \mathbf{1 0 \mathbf { r }}
\end{aligned}
$$

La même conclusion vient d'être étendue à l'azote par M. Schuster; dans son Mémoire il tend à démontrer :

I Que l'azote pur présente un spectre unique;

$2^{\circ}$ Que le spectre est un spectre de lignes;

$3^{\circ}$ Que le spectre à bandes cannelées, du premier ordre, appartient à des oxydes d'azote qui se forment sous l'influence de l'étincelle électrique.

Le point de départ des expériences de l'auteur est l'apparition soudaine des bandes cannelées à la place des lignes brillantes de l'azote dans une observation du spectre de lignes de ce gaz. Le tube de Geissler sur lequel il opérait s'était fissuré et de l'air s'y était introduit.

L'auteur fut donc conduit à construire des tubes à azote parfaitement débarrassés d'oxygène : il y parvint en absorbant les traces d'oxygène qui subsistent toujours à l'aide d'un fragment de sodium chauffé dans une ampoule attenant au tube. Grâce à cette précaution, le spectre de l'azote ne présente jamais de bandes cannelées : son speetre est exclusivement formé de lignes brillantes dont les longueurs d'onde sont les suivantes (exprimées en dix millionièmes de millimètre) :

$\begin{array}{rll}6288 & 5932 & 4644 \\ 6165 & 5666 & 4214 \\ 6152 & 5164 & 4184 \\ 5942 & 4894 & \end{array}$

Il est bon de rappeler que, si la nature des spectres de ces gaz purifiés est unique, il est probable que leur apparence doit varier lorsque la température ou la pression s'élève considérablement, et qu'ils tendent à la limite vers la continuité complète des radia- 
tions : les expériences de MM. Frankland et Lockyer sur le spectre de l'hydrogène ont prouvé que les trois lignes brillantes s'élargissent de plus en plus, et que, quand la température et la pression sont suffisantes, l'étalement des lignes est complet et le spectre devient continu.

A. Cornu. 